\title{
Biomarkers of Response to Combinational Use of PD-1 Blockade and Anti-angiogenesis: An Analysis from a Phase II Trial in Advanced Triple-negative Breast Cancer Patients
}

\author{
Jieqiong Liu \\ Sun Yat-sen memorial hospital \\ Ying Li \\ Sun Yat-sen memorial hospital \\ Qian Li \\ Sun Yat-sen memorial hospital \\ Dandan Liang
}

Genecast Precision Medicine Technology Institute

Quanren Wang

Jiangsu Hengrui Medicine Co Ltd

Qiang Liu ( $\nabla$ victorlq@hotmail.com )

Sun Yat-sen memorial hospital https://orcid.org/0000-0002-5451-4862

Research article

Keywords: biomarker analysis, PD-1 blockade, anti-angiogenesis

Posted Date: August 12th, 2020

DOI: https://doi.org/10.21203/rs.3.rs-57690/v1

License: (a) (i) This work is licensed under a Creative Commons Attribution 4.0 International License.

Read Full License 


\section{Abstract}

Background: We recently reported the results of a phase II clinical trial that the combinational use of camrelizumab plus apatinib induced an objective response rate (ORR) at $43.3 \%$ in advanced triplenegative breast cancer (TNBC) patients. This study presents the analysis of potential tumor and blood biomarkers for the patients responded to the combinational therapy.

Methods: Stromal tumor-infiltrating lymphocytes (TILs), $C D 8^{+} \mathrm{T}$ cells and the protein expression of programmed death protein 1 (PD-1) and PD-L1 were evaluated in tumor samples collected before and after the treatment. Peripheral blood samples were collected before treatment, 2-weeks and 8-weeks after treatment. 59 cytokines/chemokines, growth factors, or checkpoint-related proteins, blood immune cell subpopulations were analyzed in the blood samples. The correlation between biomarkers and clinical outcomes including ORR, progression-free survival (PFS), and overall survival (OS) was analyzed.

Results: Upon database lock, the ORR of 28 evaluable patients was $46.4 \%$. An increase of tumorinfiltrating $\mathrm{CD}^{+} \mathrm{T}$ cells more than $15 \%$ during therapy was significantly associated with higher ORR $(P=0.040)$. Patients with lower baseline plasma levels of HGF or IL-8 were more likely to respond to the combinational treatment ( $P=0.005$ or 0.001 , respectively), and showed a longer PFS and OS (HGF: $P_{\mathrm{PFS}}<0.0001, P_{\mathrm{OS}}<0.0001$; IL-8: $\left.P_{\mathrm{PFS}}<0.0001, P_{\mathrm{OS}}=0.009\right)$. Patients with a decrease of IL-8, or an increase of TIM-3 or CD152 during treatment responded more to the treatment $(P=0.008,0.040$, or 0.014 , respectively). Responders had a higher baseline $\mathrm{CD} 4^{+} \mathrm{T}$ cells and $\mathrm{B}$ cell proportions in blood than nonresponders ( $P=0.002$ and 0.030 respectively). Moreover, patients with higher baseline $\mathrm{CD} 4^{+} \mathrm{T}$ cells or $\mathrm{B}$ cells proportions in blood showed a longer PFS $(P<0.001)$ or a longer OS $(P=0.030)$ respectively.

Conclusion: Higher baseline TILs or a greater increase of tumor-infiltrating CD ${ }^{+} \mathrm{T}$ cells during therapy, lower baseline plasma HGF/IL-8, a decrease of plasma IL-8, an increase of plasma TIM-3/CD152 during therapy, higher baseline $\mathrm{CD} 4^{+} \mathrm{T}$ cells or $\mathrm{B}$ cells proportion in blood are potential biomarkers for the combinational anti-angiogenesis and immunotherapy in advanced TNBC patients.

\section{Background}

TNBC has the worst prognosis among all subtypes of breast cancer(1-4). Chemotherapy is the main systemic therapy for advanced TNBC, but drug resistance occurs rapidly and the overall survival of advanced TNBC patients is as poor as $\sim 15$ months $(5,6)$. Cancer immunotherapy has been a promising strategy in multiple kinds of solid tumors including breast cancer recently. Blockade of PD-1 or PD-L1 is considered as one of the most effective treatments of cancer immunotherapy. However, monotherapy of PD-1/PD-L1 blockade in advanced TNBC only showed marginal effect, with the ORR ranging from 5.2$18.5 \%(7-9)$. Therefore, exploring combinational strategies with other treatments to enhance the efficacy of checkpoint inhibitors is urgently needed. Our phase 2 trial of camrelizumab (an anti-PD- 1 antibody) with apatinib (a VEGFR2 inhibitor) in advanced TNBC patients is the first trial reporting the efficacy and safety of an anti-PD-1 antibody combined with an anti-angiogenesis agent in treating advanced TNBC 
patients(10). We showed that the combinational use of camrelizumab with apatinib achieved an impressively high ORR (43.3\%) with tolerable toxicities(10).

Prior trials on PD-1/PD-L1 blockade monotherapy or combination with chemotherapy in advanced breast cancer demonstrated that response to immunotherapy varies significantly among patients $(7-9,11,12)$. The fact that majority of TNBC patients did not benefit from immunotherapy indicates the pressing need to identify biomarkers that could be used to select patients that are more likely to respond to immunotherapy. A phase I trial of atezolizumab monotherapy in metastatic TNBC patients found that improved clinical activity was associated with higher levels of PD-L1-positive immune cells (ICs), CD8positive T cells and TILs(7), while another phase $1 \mathrm{~b}$ trial (KEYNOTE-012) on pembrolizumab monotherapy in metastatic TNBC reported that patients with PD-L1-positive expression on immune cells or tumor cells (TCs) may benefit more from PD-1 blockade(8). The phase 3 IMpassion130 trial showed that only advanced TNBC patients with PD-L1-positive expression on ICs had PFS and OS benefit from atezolizumab plus nab-paclitaxel $(9,12)$. However, the neoadjuvant KEYNOTE-522 phase 3 trial showed that the expression of PD-L1 level was not associated with the benefit of adding PD-1 inhibitor into chemotherapy in early TNBC patients received neoadjuvant treatment(13). Our recent report also showed that PD-L1 expression in TCs or ICs was not associated with ORR(10). These results suggest that the specific biomarkers of benefit from PD-1/PD-L1 blockade in TNBC patients remain unclear. Here we prospectively collected the tumor and blood samples in our phase 2 trial and explored potential biomarkers that can predict the response to the combinational therapy of camrelizumab with apatinib in treating advanced TNBC patients.

\section{Materials And Methods}

\subsection{Study Design and Patients}

The design and conduct of this phase 2 trial was reported previously(10). Briefly, it was an open-label, randomized, parallel, non-comparative, two-arms, phase II trial (ClinicalTrials.gov identifier:

NCT03394287). Key eligibility criteria were: female patients with age of 18-70 years with metastatic or unresectable recurrent TNBC (ER/PR-negative was defined as an ER/PR stain of $<1 \%$, HER2/Neu-negative was defined as immunohistochemistry (IHC) $0-1+$; and HER2/Neu negative by chromogenic/fluorescent in situ hybridization (FISH)); with measurable disease per Response Evaluation Criteria in Solid Tumors (RECIST) v1.1; an Eastern Cooperative Oncology Group performance status of 0/1; and received < 3 lines of systemic therapies for advanced diseases. Patients with any history of autoimmune disease; metastasis to central nervous system with clinical symptoms; any active infection or recent treatment with a systemic immunostimulatory agent (within the previous 4 weeks); recent application of systemic glucocorticoid or immunosuppressive medication; a history of using anti-angiogenesis agents or checkpoint inhibitors, or a history of severe allergic reaction to other monoclonal antibodies, or with uncontrollable hypertension or antihypertensive medication, or heart condition/disease were ineligible.

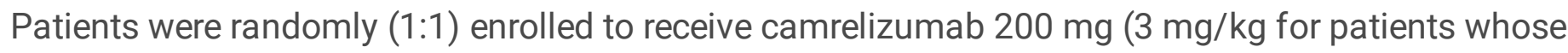
weight was below $50 \mathrm{~kg}$ ) iv, Q2W, in combination with apatinib $250 \mathrm{mg} \mathrm{po}$, continuous dosing (d1-d14), or 
intermittent dosing (d1-d7) at the first stage of this trial. Since no patient achieved response in the apatinib intermittent dosing cohort at the first stage, we only enrolled patients into the apatinib continuous dosing cohort at the second stage. Patients received treatment until unacceptable toxicities or withdrawal of consent or disease progression.

\subsection{Pathologic assessment, blood cytokine array and immune cell subpopulations assessment}

All the biomarker analyses were based on data in the apatinib continuous dosing cohort of this trial $(\mathrm{n}=$ 30). Tumor samples from metastases or recurrent tumors were collected before treatment and 8 weeks after treatment (number of paired samples: $n=20$ ). PD-L1 expression was measured using the FDAcleared 22C3 assay on the Dako Link 48 platform. The positive threshold of PD-L1 on ICs/TCs was set at $\geq 1 \%$ PD-L1-expressing immune/tumor cells as percentage of tumor area. TILs were evaluated following the criteria proposed by the International TIL WG(14). CD8 immunofluorescence and PD-1 immunohistochemistry assay were performed on tumor slides using anti-human CD8 (Cat\# MA5-14548, Thermo Fisher; 1:200) and anti-human PD-1 (Cat\# ab52587, Abcam; 1:50) antibody, respectively.

Peripheral blood samples from patients were collected in three time points: before treatment (baseline), 2 weeks after treatment, and 8 weeks after treatment. Peripheral blood samples were obtained by $10 \mathrm{ml}$ venipunctures and centrifuged $(1000 \mathrm{~g}) 15 \mathrm{~min}$ to isolate the plasma. 59 cytokines /chemokines, growth factors, or soluble checkpoint-related proteins were assessed in these plasma samples using the ProcartaPlexHuman Cytokine/Chemokine/Growth Factor Panel and the ProcartaPlexHuman ImmunoOncology Checkpoint Panel (Affymatrix, Inc). 59 cytokines /chemokines, growth factors, or soluble checkpoint-related proteins were listed in Supplementary Table 1. Details of this assay were described previously(15).

For T cell subsets or NK cell detection, $50 \mu \mathrm{l}$ peripheral blood was stained with PerCP-conjugated antiCD3, FITC-conjugated anti-CD4/PE-conjugated anti-CD8 or with FITC-conjugated anti-CD3, PE-conjugated anti-CD16/anti-CD56 and PerCP-conjugated anti-CD45 for 30 min at $4{ }^{\circ} \mathrm{C}$, respectively. For Tregs detection, whole blood was stained with FITC-conjugated anti-CD4, PC5-conjugated anti-CD25 and PEconjugated anti-CD 127 for $45 \mathrm{~min}$ at $4^{\circ} \mathrm{C}$. After antibody staining, hemolysin was used to lyse RBC. Single cell suspension was washed and then resuspended with $200 \mu \mathrm{l}$ staining buffer for flow cytometry. Flow cytometry was performed and analyzed on FACS Diva (BD bioscience). Immune cell subpopulations were defined as follows: $\mathrm{CD} 4^{+} \mathrm{T}$ cells $\left(\mathrm{CD} 3^{+} \mathrm{CD} 4^{+}\right), \mathrm{CD} 8^{+} \mathrm{T}$ cells $\left(\mathrm{CD} 3^{+} \mathrm{CD} 8^{+}\right)$, NK cells $\left(\mathrm{CD} 3^{-} \mathrm{CD} 45^{+} \mathrm{CD} 16^{+} \mathrm{CD} 56^{+}\right)$, Tregs $\left(\mathrm{CD} 4^{+} \mathrm{CD} 25^{+} \mathrm{CD} 127^{+}\right)$and $\mathrm{B}$ cells $\left(\mathrm{CD} 19^{+}\right)$. All antibodies were purchased from Beckman Coulter. Further, to detect PD $-1^{+} \mathrm{CD} 4^{+} / \mathrm{CD} 8^{+} \mathrm{T}$ cells and PD- $1^{+} \mathrm{B}$ cells, $100 \mu \mathrm{l}$ peripheral blood was stained with FITC-conjugated anti-CD3, BV605-conjugated anti-CD19, BUV395conjugated anti-CD4, BV786-conjugated anti-CD8, and BV421-conjugated anti-CD279 (PD-1) for 30 min at $4^{\circ} \mathrm{C}$. Then lysing RBC was performed by using lysing buffer. After lysing, cells were washed and then resuspended to single cell suspension with $200 \mu$ staining buffer. Flow cytometry was performed on FACS Celesta (BD bioscience) and was analyzed with FlowJo software. Immune cell subpopulations were 
defined as follows: $\mathrm{CD} 4^{+} \mathrm{PD}-1^{+} \mathrm{T}$ cells $\left(\mathrm{CD} 3^{+} \mathrm{CD} 4^{+} \mathrm{CD} 8^{-} \mathrm{PD}-1^{+}\right), \mathrm{CD} 8^{+} \mathrm{PD}-1^{+} \mathrm{T}$ cells $\left(\mathrm{CD} 3^{+} \mathrm{CD} 4^{-} \mathrm{CD} 8^{+} \mathrm{PD}-1^{+}\right)$, and $\mathrm{PD}-1^{+} \mathrm{B}$ cells $\left(\mathrm{CD} 19^{+} \mathrm{PD}-1^{+}\right)$. All these antibodies were purchased from BD bioscience.

\subsection{End point and statistical analysis}

The primary end point of this study was correlations among biomarkers and patient outcomes including ORR, PFS, and OS in the apatinib continuous dosing cohort. PFS, and OS were evaluated by the KaplanMeier method. Log-rank test and chi square test were used to assess associations between biomarkers and outcomes. Mann-Whitney $U$ test was used to assess associations between plasma cytokines/chemokines/growth factors and outcomes or TRAE. Statistical analyses were performed using STATA (v13.0) and R software packages. All statistical tests were two-sided, with statistical significance defined as $\mathrm{P}<0.05$.

\section{Results}

\subsection{Patients and Treatment}

From January 2018 to April 2019, 40 TNBC patients received 0-2 prior lines of systemic therapies for advanced/metastatic diseases at Sun Yat-sen Memorial Hospital were enrolled into the phase II trial of camrelizumab with apatinib, including 10 in the apatinib intermittent dosing cohort, and 30 in the apatinib continuous dosing cohort. All the biomarker analyses were based on data of the 30 patients in the continuous apatinib dosing cohort of this trial. 28 of 30 patients were evaluable for overall response. Baseline characteristics of these 28 patients were listed in Table 1. Upon database lock in this study (March 4th, 2020), the median follow-up duration was 10.2 (range 2.1-25.2) months. The ORR of these 28 evaluable patients was $46.4 \%$ (13/28, 95\% Cl: 27.5\%-66.1\%). 
Table 1

Baseline patient characteristics

\begin{tabular}{|ll|}
\hline & $\begin{array}{l}\text { Continuous dosing } \\
\text { cohort }(\mathbf{n}=\mathbf{2 8})\end{array}$ \\
\hline Age, years, median (range) & $46(32-64)$ \\
\hline Prior therapy in advanced setting & \\
\hline No & $7(25.0)$ \\
\hline 1 line & $15(53.6)$ \\
\hline 2 lines & $6(21.4)$ \\
\hline Metastatic disease & $27(96.4)$ \\
\hline No. of sites of metastatic disease & \\
\hline $0-3$ & $21(75.0)$ \\
\hline$\geq 4$ & $7(25.0)$ \\
\hline Site of metastatic disease & \\
\hline Bone & $6(21.4)$ \\
\hline Live & $8(28.6)$ \\
\hline Lung & $12(42.9)$ \\
\hline Lymph node only & $2(7.1)$ \\
\hline PD-L1 IC status & $17(10.7)$ \\
\hline Negative & $10(35.7)$ \\
\hline Positive & $1(39.3)$ \\
\hline PD-L1 TC status & \\
\hline Negative & \\
\hline Positive & \\
\hline BRCA1/2 mutation & \\
\hline Negative & \\
\hline Positive & \\
\hline
\end{tabular}

Data presented as $\mathrm{n}(\%)$, or median (range). IC $=$ immune cells. $\mathrm{TC}=$ tumor cells.

${ }^{1}$ Some patients had multiple sites of metastatic disease. 


\begin{tabular}{|ll|}
\hline & \multicolumn{1}{|c|}{$\begin{array}{l}\text { Continuous dosing } \\
\text { cohort }(\mathbf{n = 2 8 )}\end{array}$} \\
\hline Unknown & $2(7.1)$ \\
\hline Data presented as $\mathrm{n}(\%)$, or median (range). IC = immune cells. TC = tumor cells. \\
\hline 1 Some patients had multiple sites of metastatic disease.
\end{tabular}

\subsection{Tumor tissue biomarker assessment}

In the previous report(10), we showed that the PD-L1 expression on ICs and TCs before therapy was not correlated with ORR or PFS. The infiltration of $\mathrm{CD} 8^{+} \mathrm{T}$ cells and PD-1 expression on ICs before therapy also did not correlate with clinical outcomes. Nevertheless, a high percentage of baseline TILs (>10\%) was associated with higher ORR and favorable PFS ( $P=0.029$ and 0.054 , respectively)(10).

In this study, the updated follow-up data were used to explore the correlations between clinical outcomes and immune parameters in tumor including PD-L1 expression on TCs or ICs, stromal TILs and CD8 ${ }^{+} \mathrm{T}$ cells, and PD-1 expression on immune cells. A high percentage of baseline TILs (>10\%) was still associated with longer PFS ( $P=0.035$, Figure $\mathrm{S} 1)$. No significant correlation between clinical outcomes and baseline PD-L1 expression on ICs/TCs or PD-1 expression on ICs was observed ( $P>0.05$ for all)(10). Moreover, we explored the associations between clinical outcomes and changes of these immune parameters at eight weeks after treatment. A greater increase (>15\%) of tumor-infiltrating $\mathrm{CD}^{+} \mathrm{T}^{\mathrm{T}}$ cells at eight weeks after treatment was associated with ORR (Fig. 1, P = 0.040), but not with longer PFS or OS ( $P$ $=0.181$, and 0.563 , respectively). There was no correlation between clinical outcomes and changes of PDL1 expression on ICs/TCs, TILs or PD-1 expression on ICs at eight weeks after treatment ( $P>0.05$ for all), indicating the response to this combinational immunotherapy is independent of the expression of PDL1/PD-1 in tumor.

\subsection{Peripheral blood biomarker assessment}

We evaluated the association between germline BRCA1/2 status and ORR. Among the 28 evaluable patients, 26 patients have known germline BRCA1/2 status, with two BRCA1 mutations and one BRCA2 mutation. There was no statically significant correlation between germline BRCA $1 / 2$ status and ORR $(P=$ 0.449).

Using the ProcartaPlexHuman Cytokine/Chemokine/Growth Factor Panel and the Immuno-Oncology Checkpoint Panel assay, the peripheral blood samples from 25 evaluable patients were measured for the amount of 59 cytokines/chemokines, growth factors, or soluble checkpoint-related proteins. The baseline plasma levels of HGF and IL-8 were significantly lower in responders (partial response, PR) than in nonresponders (stable disease, SD or progressive disease, PD) (HGF: $74.37 \mathrm{pg} / \mathrm{ml} v s .110 .99 \mathrm{pg} / \mathrm{ml}, P=$ 0.005; IL-8: 0 vs. 2.15 pg/ml, $P=0.001$; Fig. 2a and 2b). Patients with lower baseline HGF or IL-8 showed a significantly longer PFS and OS compared with those with higher baseline HGF or IL-8 (HGF: 
$P_{\mathrm{PFS}}<0.0001, P_{\mathrm{OS}}<0.0001 ;$ IL-8: $P_{\mathrm{PFS}}<0.0001, P_{\mathrm{OS}}=0.009 ;$ Fig. 2c-2f). Moreover, the fold changes of plasma IL-8, NGF-beta, FGF-2, TIM-3 and CD152 at 8 weeks after treatment from baseline were significantly correlated with the response to camrelizumab plus apatinib therapy $(P=0.008,0.019,0.005,0.040$, and 0.014 , respectively, Fig. 3).

Next, we assessed the correlations between baseline plasma protein levels and incidences of different treatment-related adverse effects (TRAE). Details of TRAE of the enrolled patients were reported previously(10). We divided TRAE into 10 categories, including hematological, neuropsychiatric, skin, gastrointestinal, urinary, cardiovascular, hemorrhagic, respiratory, endocrine, and other adverse effects. We observed that the patients with lower baseline plasma IL-18, or IFN-y levels were more likely to suffer from gastrointestinal TRAEs ( $P=0.007$ and 0.031 , respectively, Fig. $4 \mathrm{a}$ and $4 \mathrm{~b})$, whereas patients with higher baseline plasma VEGF-A or MIP-1 3 were more likely to have respiratory TRAEs $(P=0.043$ and 0.049 , respectively, Fig. $4 \mathrm{c}$ and $4 d$ ). Additionally, patients with higher baseline plasma TIM-3, IL-18, IFN- $y$, CD152, or CD28 levels were more likely to suffer from skin side effects $(P=0.025,0.014,0.021,0.030$ and 0.043 , respectively, Fig. 4e-4i).

The immune cell subpopulations in peripheral blood were examined at baseline for all 28 evaluable patients, and at both 2 and 8 weeks after treatment for 26 of them. The proportions of CD $4^{+} \mathrm{T}$ cells in blood decreased significantly at 2 and 8 weeks after treatment compared with that at baseline $(P=0.035$, 0.018 , respectively, Figure S2a). All PR patients displayed a $\mathrm{CD} 4^{+} \mathrm{T}$ cells percentage $>31 \%$ at baseline, while only $46.7 \%$ of SD/PD patients displayed a CD $4^{+}$T cells percentage $>31 \%$ at baseline $(P=0.002$, Fig. 5a). Moreover, the patients with higher $\mathrm{CD} 4^{+} \mathrm{T}$ cells proportions at baseline showed a significantly longer PFS than those with lower proportions of CD $4^{+}$T cells (5.9 vs. 2.0 months, $P<0.001$, Fig. 5b), although the difference of OS was not statistically significant (11.3 vs. 5.9 months, $P=0.646$, Figure $\mathrm{S} 2 \mathrm{~b})$. The proportion of B cells in blood significantly decreased at 2 weeks after treatment compared with that at baseline ( $P<0.001$, Figure S2C). $76.9 \%$ of PR patients displayed a B cells percentage $>13 \%$ at baseline, while $33.3 \%$ of SD/PD patients displayed a B cells percentage $>13 \%$ at baseline $(P=0.030$, Fig. 5c). Patients with higher baseline B cells proportions showed a statistically longer OS than those with lower baseline proportions of B cells (not reached vs. 4.6 months, $P=0.030$, Fig. $5 \mathrm{~d}$ ), although the difference of PFS was not statistically significant (5.9 vs. 3.0 months, $P=0.126$, Figure S2d). Together, these results indicate that higher percentage of $C D 4^{+} T$ cells or $B$ cells in blood at baseline could serve as biomarkers to predict the response to combinational immunotherapy and better prognosis.

The proportion of NK cells in blood significantly increased at 2 weeks after treatment compared with that at baseline ( $P=0.003$, Figure S3a). However, there were no significant correlations between the response and the baseline proportions of NK cells, Tregs and CD8 $8^{+}$cells $(P>0.05$ for all).

To explore the impact of PD-1 expression on immune cells, the subpopulations of CD $4^{+} \mathrm{PD}-1^{+} \mathrm{T}$ cell, CD8 ${ }^{+} \mathrm{PD}-1^{+} \mathrm{T}$ cell and PD- $1^{+} \mathrm{B}$ cell was measured in 25 evaluable patients. The proportions of $\mathrm{CD} 4^{+} \mathrm{PD}-1^{+}$ $T$ cells or $C D 8^{+} \mathrm{PD}-1^{+} \mathrm{T}$ cells at 2 or 8 weeks after treatment significantly decreased compared with those 
at baseline $\left(P<0.001\right.$, Figure S3b-3c). Nevertheless, none of the baseline proportions of CD $4^{+} \mathrm{PD}-1^{+} \mathrm{T}$ cells, CD $8^{+} \mathrm{PD}-1^{+} \mathrm{T}$ cells and PD-1 $1^{+} \mathrm{B}$ cells was found to be associated with the response to combinational immunotherapy ( $P>0.05$ for all). Additionally, the response to immunotherapy was not associated with the fold changes of any immune cell subpopulations at 2 or 8 weeks after treatment from baseline $(P>0.05$ for all).

\section{Discussion}

In this study, we examined several tissue and blood markers and explored their association with clinical response to a combinational therapy of immune checkpoint blockade and anti-angiogenesis in treating advanced TNBC patients. We have demonstrated that advanced TNBC patients with more baseline TILs or a greater increase of tumor-infiltrating $C D 8^{+} T$ cells during therapy, or with lower baseline plasma HGF or IL-8 levels, or a decrease of plasma IL-8, or an increase of plasma TIM-3 or CD152 during treatment, or with higher baseline blood $\mathrm{CD} 4^{+} \mathrm{T}$ cells or $\mathrm{B}$ cells proportion may benefit more from camrelizumab plus apatinib combinational therapy. Additionally, we observed that distinct baseline plasma proteins might predict different TRAEs during this combinational therapy.

Previous studies showed that several biomarkers such as PD-L1/PD-1 expression on TCs or ICs, and stromal TILs and CD8 ${ }^{+} T$ cells may predict the response to anti-PD-1/PD-L1 cancer therapy $(7,16)$. We reported priorly that neither baseline PD-L1 expression on ICs nor that on TCs correlated with ORR or prolonged PFS, and the infiltration of baseline CD8 ${ }^{+} \mathrm{T}$ cells, and PD-1 expression on ICs also did not correlate with outcomes; but a high percentage of baseline TILs was associated with higher ORR and favorable PFS(10). Here in this study, we found that advanced TNBC patients with more baseline TILs (> $10 \%)$ or a greater increase $(>15 \%)$ of tumor-infiltrating $C D 8^{+} T$ cells 8 weeks after treatment were more sensitive to camrelizumab plus apatinib combinational therapy. This finding is consistent with prior earlyphase trials. For instance, a phase I trial of atezolizumab monotherapy in metastatic TNBC found that improved clinical activity was associated with higher levels of tumor CD8 ${ }^{+} \mathrm{T}$ cells, and TILs(7).

Using the ProcartaPlexHuman Cytokine/Chemokine/Growth Factor Panel and the ProcartaPlexHuman Immuno-Oncology Checkpoint Panel, we found that patients with lower baseline plasma HGF or IL-8 levels, or a decrease of plasma IL-8, or an increase of plasma TIM-3 or CD152 level at 8 weeks after treatment may benefit more from camrelizumab with apatinib. Recent studies showed that high serum levels of the c-MET ligand HGF correlated with increasing neutrophil counts, which was associated with poor responses to checkpoint blockade therapies(17-20). IL-8 is secreted by malignant tumor and stroma cells across many different types of tumor, and is a member of the CXC chemokine family identified as a chemotactic factor for neutrophils(21-23). A previous study also reported that decreases in serum IL-8 level 2-4 weeks after anti-PD-1 blockade therapies were strongly associated with response to immune checkpoint blockade in metastatic melanoma and NSCLC patients(24). Furthermore, higher serum TIM-3 or CD152 (also known as CTLA-4) level may indicate a more favorable clinical outcomes in 
advanced cancer patients, and higher baseline serum TIM-3 or CD152 can predict response to immune checkpoint blockade in patients with metastatic gastrointestinal tract cancer(15, 25, 26).

Recent studies found that tumor-infiltrating B cells predict a better response to immunotherapy in several kinds of solid tumors, including melanoma, renal cell carcinoma, and sarcoma(27-29). A retrospective study found that higher baseline blood $\mathrm{CD}^{+} \mathrm{T}$ cells may predict the outcome of anti-PD- 1 treatment in patients with advanced non-small cell lung cancer(30). Another study found that blood $\mathrm{CD} 4^{+}$memory $\mathrm{T}$ cell subsets played an important role in response to anti-CTLA-4 therapy in melanoma patients(31). However, very few studies have focused on peripheral blood B cells or CD4 ${ }^{+} \mathrm{T}$ cells and their impact on the response to immune checkpoint blockade in breast cancer patients. In this study, we observed that advanced TNBC patients with higher baseline blood $\mathrm{CD} 4^{+} \mathrm{T}$ cells or $\mathrm{B}$ cells proportion may benefit more from camrelizumab plus apatinib combinational therapy. This suggests that pre-treatment blood $\mathrm{CD} 4^{+} \mathrm{T}$ cells and $\mathrm{B}$ cells proportions may be useful biomarkers for predicting potential patients who may benefit more from camrelizumab plus apatinib therapy.

The sample size in this study is small, thus the conclusions should be interpreted with caution. The biomarkers will be validated in an ongoing phase III randomized clinical trial. Additionally, we only performed tumor tissue biopsy at 8 weeks after treatment, so we cannot study the associations between ephemeral changes of tumor immune microenvironment during treatment and the treatment response.

\section{Conclusions}

In conclusion, this perspective study have assessed the baseline and dynamic change of tumor and blood biomarkers to predict the response to camrelizumab plus apatinib combinational therapy in patients with advanced TNBC. We found that more baseline TILs or a greater increase of tumorinfiltrating $\mathrm{CD}^{+}{ }^{+} \mathrm{T}$ cells during therapy, or lower baseline plasma HGF/IL-8, or a decrease of plasma IL-8, or an increase of plasma TIM-3/CD152 during therapy, or higher baseline blood CD $4^{+} T$ cells or $B$ cells proportion may identify responders to camrelizumab plus apatinib combinational therapy. Further perspective studies with larger sample size are needed to confirm these findings.

\section{Abbreviations}

\section{ORR}

objective response rate

TNBC

triple-negative breast cancer

TILs

tumor-infiltrating lymphocytes

PD-1

programmed death protein 1

PFS 
progression-free survival

OS

overall survival

ICs

immune cells

TCs

tumor cells

PR

partial response

SD

stable disease

PD

progressive disease

TRAE

treatment-related adverse effects

\section{Declarations}

\section{Author Contributions}

Conceptualization, Qiang Liu; Formal analysis, Jieqiong Liu, Ying Li and Qian Li; Methodology, Jieqiong Liu, Ying Li and Qian Li; Project administration, Qiang Liu; Writing - original draft, Jieqiong Liu, Ying Li and Qian Li; Writing - review \& editing, Jieqiong Liu, Ying Li, Qian Li, Dandan Liang, Quanren Wang and Qiang Liu.

\section{Ethics approval and consent to participate}

This research was approved by the Clinical Research Ethics Committee of Sun Yat-sen Memorial Hospital of Sun Yat-sen University. And all the studies were performed in accordance with the International Ethical Guidelines for Biomedical Research Involving Human Subjects (CIOMS). Written informed consents were obtained from the participants before sampling.

\section{Funding}

This work was supported by a grant from the Fundamental Research Funds for the Central Universities (20ykpy101).

\section{Acknowledgments}


We thank the patients and their families, nurses, trial coordinators, radiologists and pathologists who participated in this trial.

\section{Competing interests}

The authors declare no conflicts of interest.

\section{Consent for publication}

Not applicable.

\section{Availability of data and materials}

The datasets used and/or analyzed during the current study are available from the corresponding author on reasonable request

\section{References}

1. Li X, Yang J, Peng L, Sahin AA, Huo L, Ward KC, et al. Triple-negative breast cancer has worse overall survival and cause-specific survival than non-triple-negative breast cancer. Breast Cancer Res Treat. 2017;161(2):279-87.

2. He X, Ji J, Dong R, Liu H, Dai X, Wang C, et al. Prognosis in different subtypes of metaplastic breast cancer: a population-based analysis. Breast Cancer Res Treat. 2019;173(2):329-41.

3. Malorni L, Shetty PB, De Angelis C, Hilsenbeck S, Rimawi MF, Elledge R, et al. Clinical and biologic features of triple-negative breast cancers in a large cohort of patients with long-term follow-up. Breast Cancer Res Treat. 2012;136(3):795-804.

4. Metzger-Filho O, Sun Z, Viale G, Price KN, Crivellari D, Snyder RD, et al. Patterns of Recurrence and outcome according to breast cancer subtypes in lymph node-negative disease: results from international breast cancer study group trials VIII and IX. J Clin Oncol. 2013;31(25):3083-90.

5. den Brok WD, Speers CH, Gondara L, Baxter E, Tyldesley SK, Lohrisch CA. Survival with metastatic breast cancer based on initial presentation, de novo versus relapsed. Breast Cancer Res Treat. 2017;161(3):549-56.

6. Gobbini E, Ezzalfani M, Dieras V, Bachelot T, Brain E, Debled M, et al. Time trends of overall survival among metastatic breast cancer patients in the real-life ESME cohort. Eur J Cancer. 2018;96:17-24.

7. Emens LA, Cruz C, Eder JP, Braiteh F, Chung C, Tolaney SM, et al. Long-term Clinical Outcomes and Biomarker Analyses of Atezolizumab Therapy for Patients With Metastatic Triple-Negative Breast Cancer: A Phase 1 Study. JAMA Oncol. 2019;5(1):74-82. 
8. Nanda R, Chow LQ, Dees EC, Berger R, Gupta S, Geva R, et al. Pembrolizumab in Patients With Advanced Triple-Negative Breast Cancer: Phase Ib KEYNOTE-012 Study. J Clin Oncol. 2016;34(21):2460-7.

9. Dirix LY, Takacs I, Jerusalem G, Nikolinakos P, Arkenau HT, Forero-Torres A, et al. Avelumab, an antiPD-L1 antibody, in patients with locally advanced or metastatic breast cancer: a phase 1b JAVELIN Solid Tumor study. Breast Cancer Res Treat. 2018;167(3):671-86.

10. Liu J, Liu Q, Li Y, Li Q, Su F, Yao H, et al. Efficacy and safety of camrelizumab combined with apatinib in advanced triple-negative breast cancer: an open-label phase II trial. J Immunother Cancer. 2020;8(1).

11. Schmid P, Adams S, Rugo HS, Schneeweiss A, Barrios $\mathrm{CH}$, Iwata $\mathrm{H}$, et al. Atezolizumab and NabPaclitaxel in Advanced Triple-Negative Breast Cancer. N Engl J Med. 2018;379(22):2108-21.

12. Schmid P, Rugo HS, Adams S, Schneeweiss A, Barrios $\mathrm{CH}$, Iwata $\mathrm{H}$, et al. Atezolizumab plus nabpaclitaxel as first-line treatment for unresectable, locally advanced or metastatic triple-negative breast cancer (IMpassion130): updated efficacy results from a randomised, double-blind, placebocontrolled, phase 3 trial. Lancet Oncol. 2020;21(1):44-59.

13. Neoadjuvant Pembrolizumab Takes on TNBC. Cancer Discov. 2019;9(10):OF4.

14. Salgado R, Denkert C, Demaria S, Sirtaine N, Klauschen F, Pruneri G, et al. The evaluation of tumorinfiltrating lymphocytes (TILs) in breast cancer: recommendations by an International TILs Working Group 2014. Ann Oncol. 2015;26(2):259-71.

15. Lu Z, Zou J, Hu Y, Li S, Zhou T, Gong J, et al. Serological Markers Associated With Response to Immune Checkpoint Blockade in Metastatic Gastrointestinal Tract Cancer. JAMA Netw Open. 2019;2(7):e197621.

16. Loi S, Giobbie-Hurder A, Gombos A, Bachelot T, Hui R, Curigliano G, et al. Pembrolizumab plus trastuzumab in trastuzumab-resistant, advanced, HER2-positive breast cancer (PANACEA): a singlearm, multicentre, phase 1b-2 trial. Lancet Oncol. 2019;20(3):371-82.

17. Glodde N, Bald T, van den Boorn-Konijnenberg D, Nakamura K, O'Donnell JS, Szczepanski S, et al. Reactive Neutrophil Responses Dependent on the Receptor Tyrosine Kinase c-MET Limit Cancer Immunotherapy. Immunity. 2017;47(4):789-802.e9.

18. Gebhardt C, Sevko A, Jiang H, Lichtenberger R, Reith M, Tarnanidis K, et al. Myeloid Cells and Related Chronic Inflammatory Factors as Novel Predictive Markers in Melanoma Treatment with Ipilimumab. Clin Cancer Res. 2015;21(24):5453-9.

19. Peng S, Wang R, Zhang X, Ma Y, Zhong L, Li K, et al. EGFR-TKI resistance promotes immune escape in lung cancer via increased PD-L1 expression. Mol Cancer. 2019;18(1):165.

20. Papaccio F, Della Corte CM, Viscardi G, Di Liello R, Esposito G, Sparano F, et al. HGF/MET and the Immune System: Relevance for Cancer Immunotherapy. Int J Mol Sci. 2018;19(11).

21. Waugh DJ, Wilson C. The interleukin-8 pathway in cancer. Clin Cancer Res. 2008;14(21):6735-41.

22. Zarogoulidis P, Katsikogianni F, Tsiouda T, Sakkas A, Katsikogiannis N, Zarogoulidis K. Interleukin-8 and interleukin-17 for cancer. Cancer Invest. 2014;32(5):197-205. 
23. Schalper KA, Carleton M, Zhou M, Chen T, Feng Y, Huang SP, et al. Elevated serum interleukin-8 is associated with enhanced intratumor neutrophils and reduced clinical benefit of immune-checkpoint inhibitors. Nat Med. 2020;26(5):688-92.

24. Sanmamed MF, Perez-Gracia JL, Schalper KA, Fusco JP, Gonzalez A, Rodriguez-Ruiz ME, et al. Changes in serum interleukin-8 (IL-8) levels reflect and predict response to anti-PD-1 treatment in melanoma and non-small-cell lung cancer patients. Ann Oncol. 2017;28(8):1988-95.

25. Anderson AC. Tim-3: an emerging target in the cancer immunotherapy landscape. Cancer Immunol Res. 2014;2(5):393-8.

26. Liu Q, Hu P, Deng G, Zhang J, Liang N, Xie J, et al. Soluble cytotoxic T-lymphocyte antigen 4: a favorable predictor in malignant tumors after therapy. Onco Targets Ther. 2017;10:2147-54.

27. Petitprez F, de Reyniès A, Keung EZ, Chen TW, Sun CM, Calderaro J, et al. B cells are associated with survival and immunotherapy response in sarcoma. Nature. 2020;577(7791):556-60.

28. Helmink BA, Reddy SM, Gao J, Zhang S, Basar R, Thakur R, et al. B cells and tertiary lymphoid structures promote immunotherapy response. Nature. 2020;577(7791):549-55.

29. Cabrita R, Lauss M, Sanna A, Donia M, Skaarup Larsen M, Mitra S, et al. Tertiary lymphoid structures improve immunotherapy and survival in melanoma. Nature. 2020;577(7791):561-5.

30. Ottonello S, Genova C, Cossu I, Fontana V, Rijavec E, Rossi G, et al. Association Between Response to Nivolumab Treatment and Peripheral Blood Lymphocyte Subsets in Patients With Non-small Cell Lung Cancer. Front Immunol. 2020;11:125.

31. Subrahmanyam PB, Dong Z, Gusenleitner D, Giobbie-Hurder A, Severgnini M, Zhou J, et al. Distinct predictive biomarker candidates for response to anti-CTLA-4 and anti-PD-1 immunotherapy in melanoma patients. J Immunother Cancer. 2018;6(1):18.

\section{Figures}




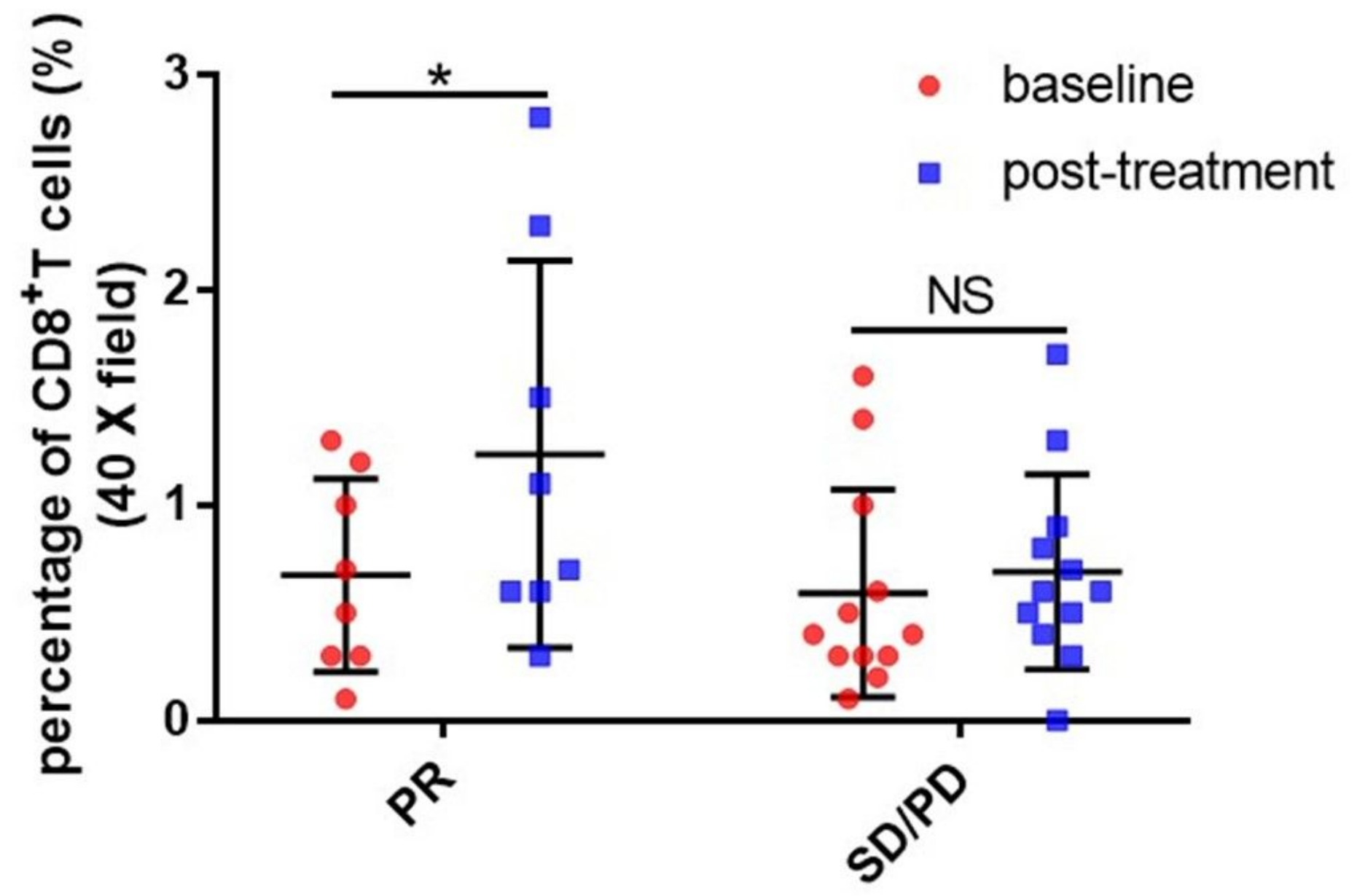

Figure 1

Association between the changes of tumor-infiltrating CD8+ T cells during therapy and the objective response rate. Changes of tumor-infiltrating $C D 8+T$ cells during therapy in PR patients, and SD/PD patients. 
a

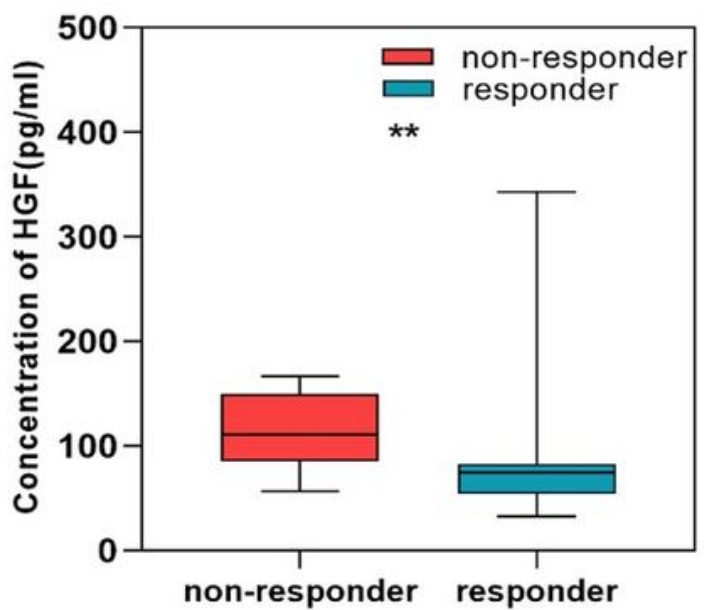

C

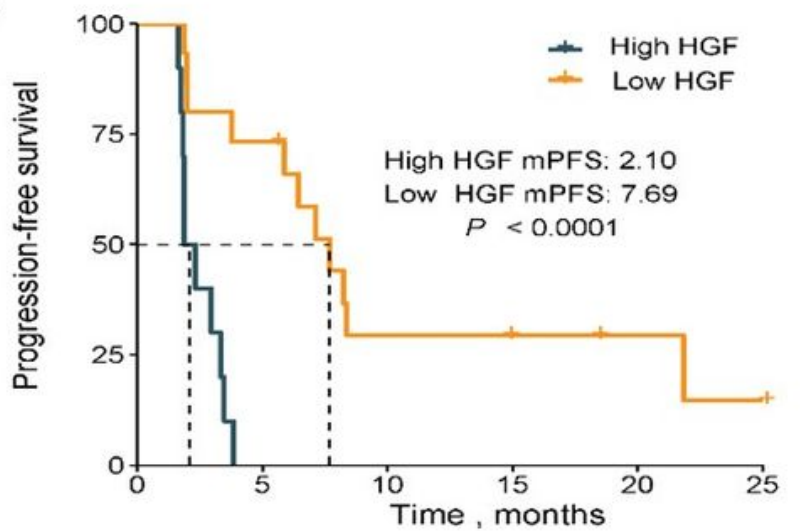

Number at risk $\begin{array}{ll}\text { High HGF } & 10 \\ \text { Low HGF } & 15\end{array}$

e

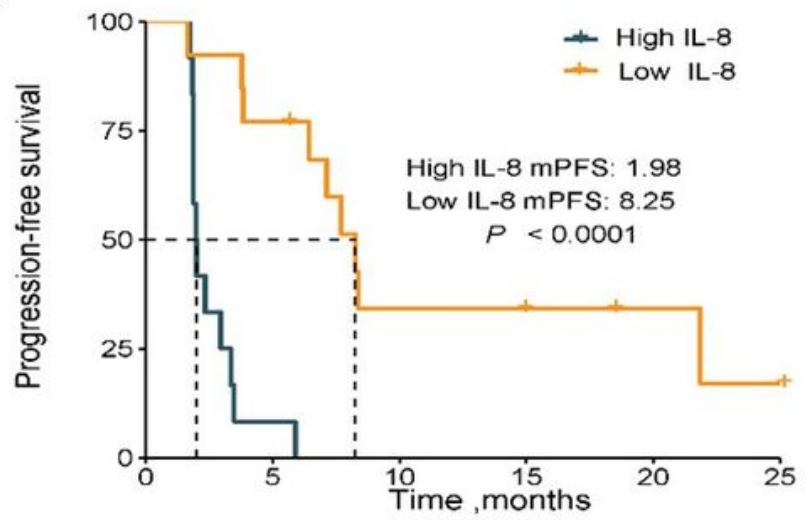

Number at risk

$\begin{array}{ccccccc}\text { High IL-8 } & 12 & 1 & 0 & 0 & 0 & 0 \\ \text { Low IL-8 } & 13 & 10 & 4 & 3 & 2 & 1\end{array}$ b

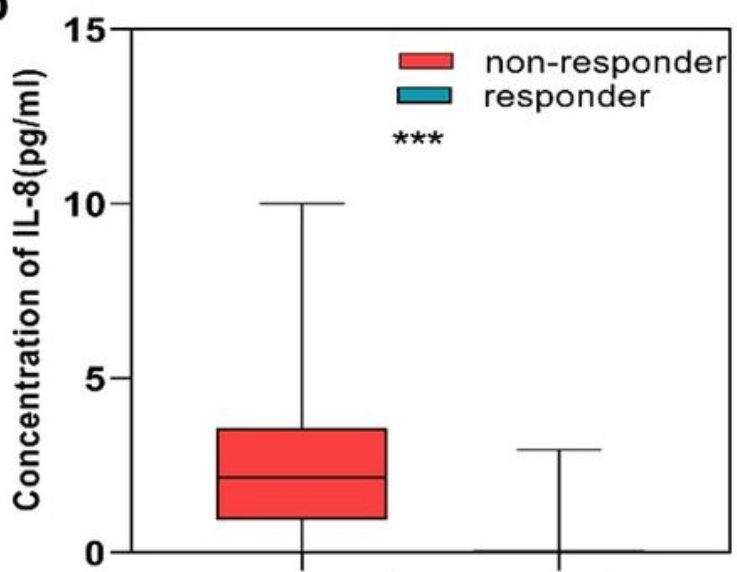

non-responder responder

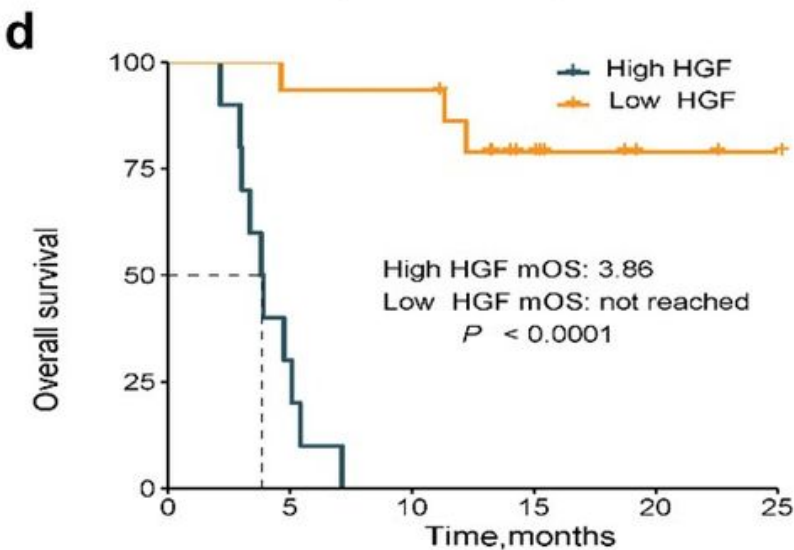

Number at risk

$\begin{array}{lcccccc}\text { High HGF } & 10 & 3 & 0 & 0 & 0 & 0 \\ \text { Low HGF } & 15 & 14 & 14 & 7 & 2 & 1\end{array}$

f

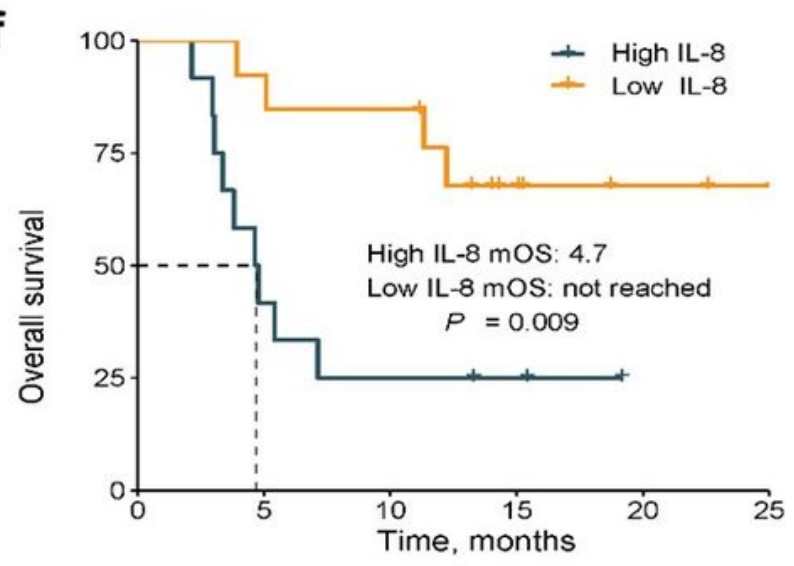

Number at risk

$\begin{array}{ccccccc}\text { High IL-8 } & 12 & 5 & 3 & 2 & 0 & 0 \\ \text { Low IL-8 } & 13 & 12 & 11 & 5 & 2 & 1\end{array}$

\section{Figure 2}

Associations of plasma proteins levels and clinical outcomes. Baseline plasma a HGF or b IL-8 levels in responders and non-responders; c Progression-free survival or d overall survival stratified by baseline plasma HGF level. e Progression-free survival or $\mathrm{f}$ overall survival stratified by baseline plasma IL-8 level. 


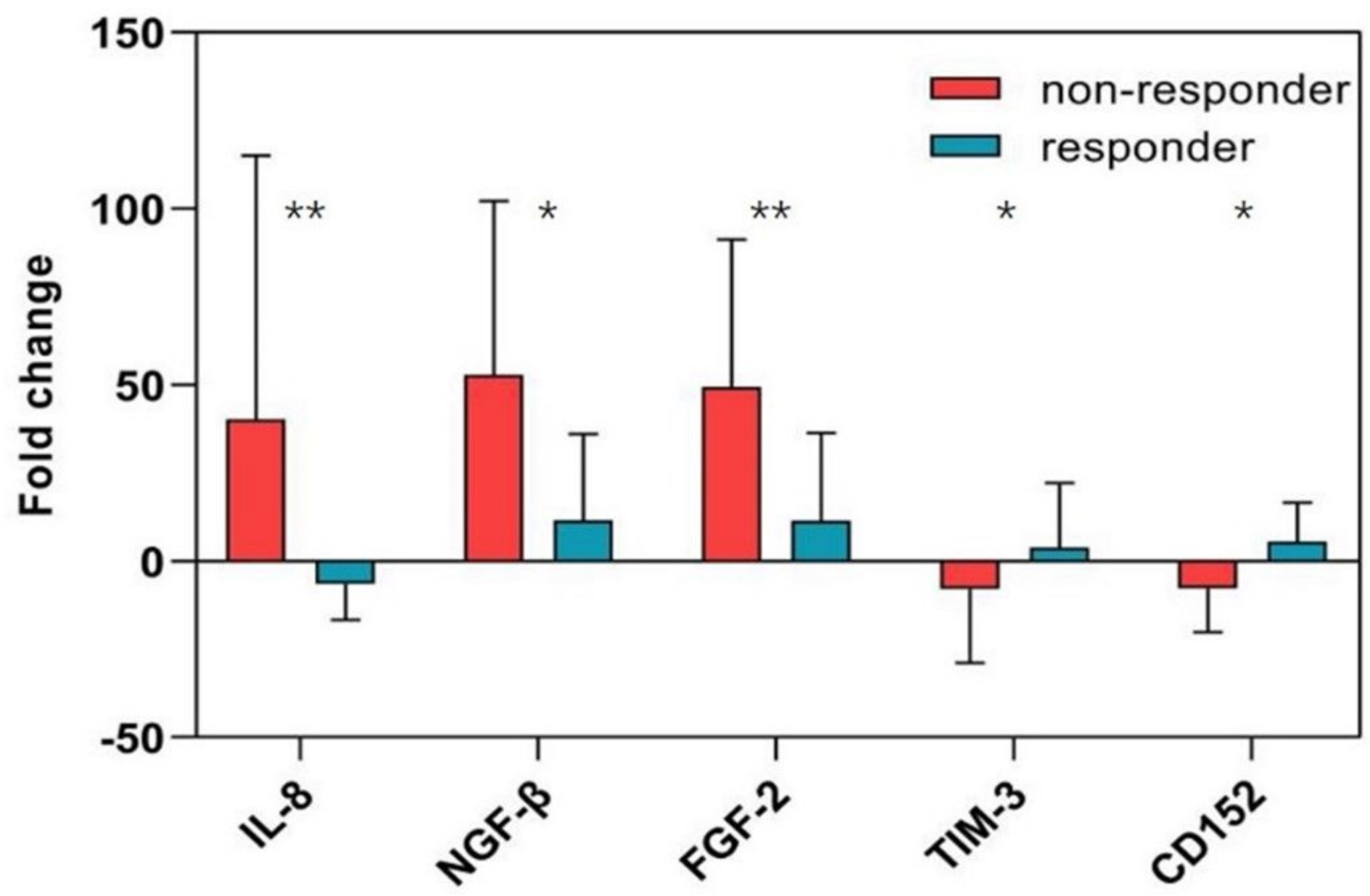

Figure 3

Fold changes of plasma IL-8, NGF-beta, FGF-2, TIM-3 and CD152 from 8 weeks after treatment to baseline in responders and non-responders. 
a

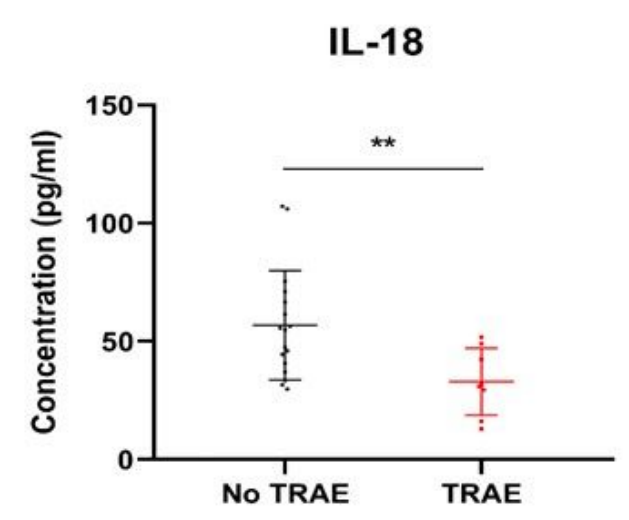

d

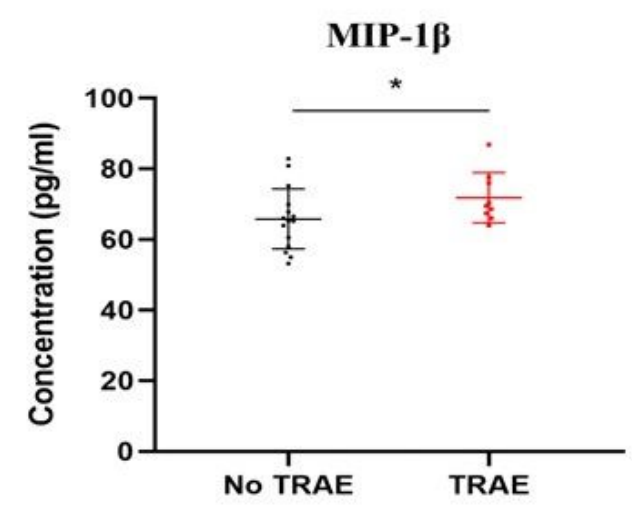

g

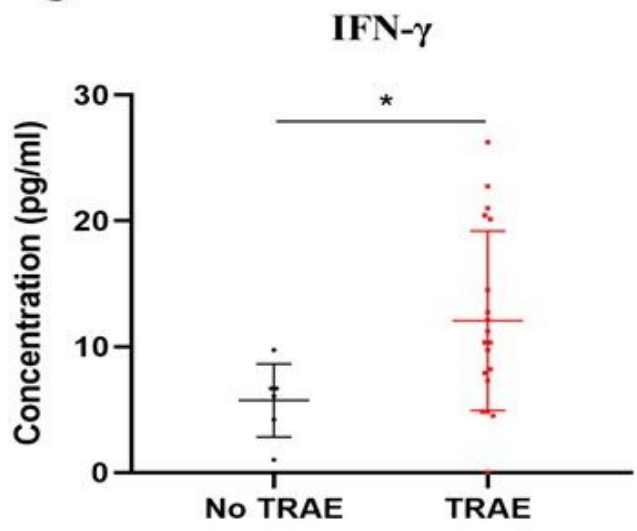

b

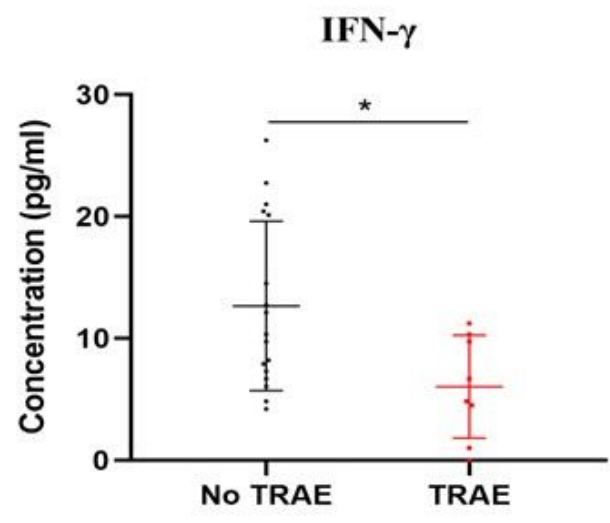

e

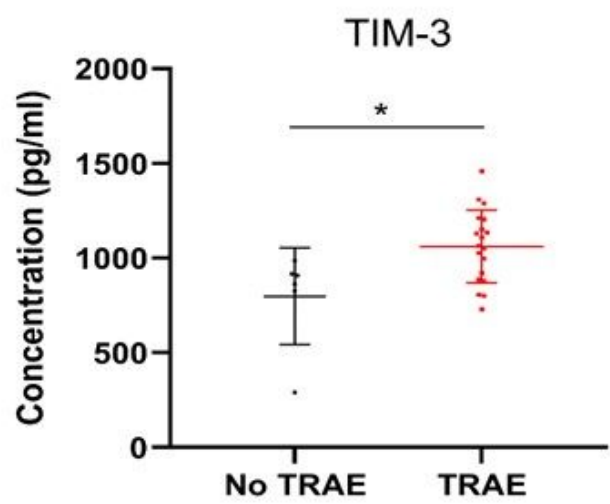

h

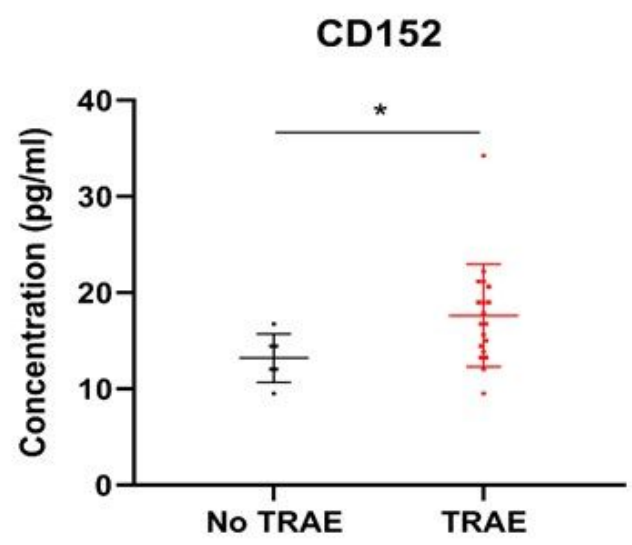

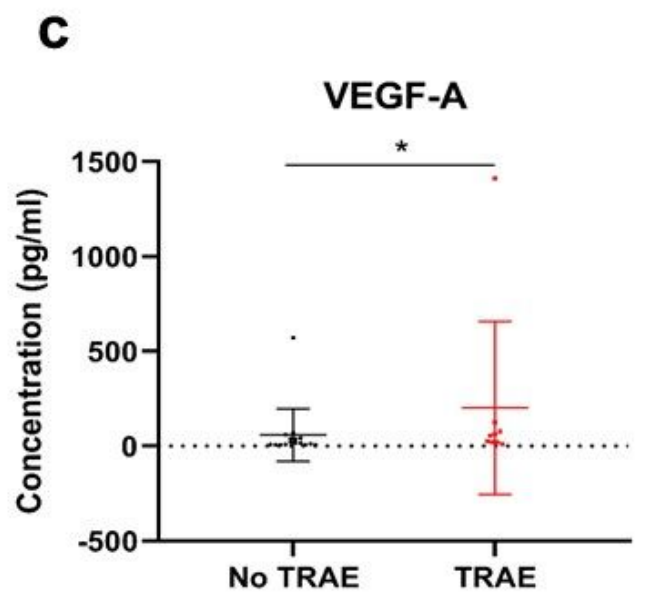

$\mathbf{f}$

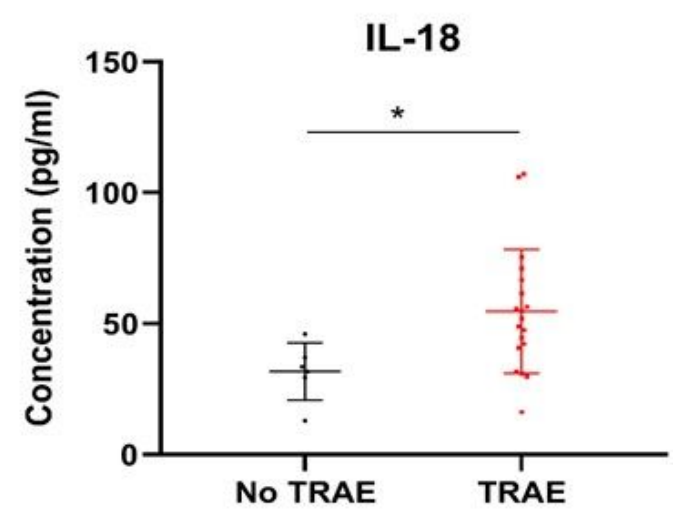

i

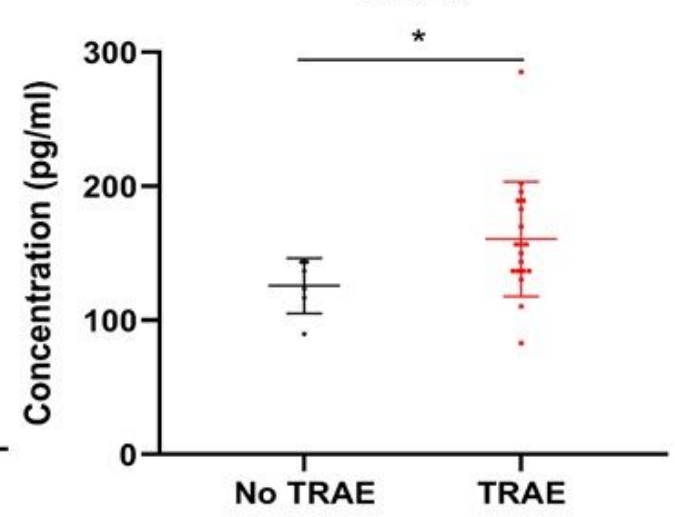

\section{Figure 4}

Associations of baseline plasma proteins levels and TRAE. Baseline plasma a IL-18 or b IFN-y level in patients with and without gastrointestinal TRAEs; Baseline plasma c VEGF-A or d MIP-1ß level in patients with and without respiratory TRAEs; Baseline plasma e TIM-3 or f IL-18 or g IFN-y or h CD152 or i CD28 level in patients with and without skin TRAEs. 
a

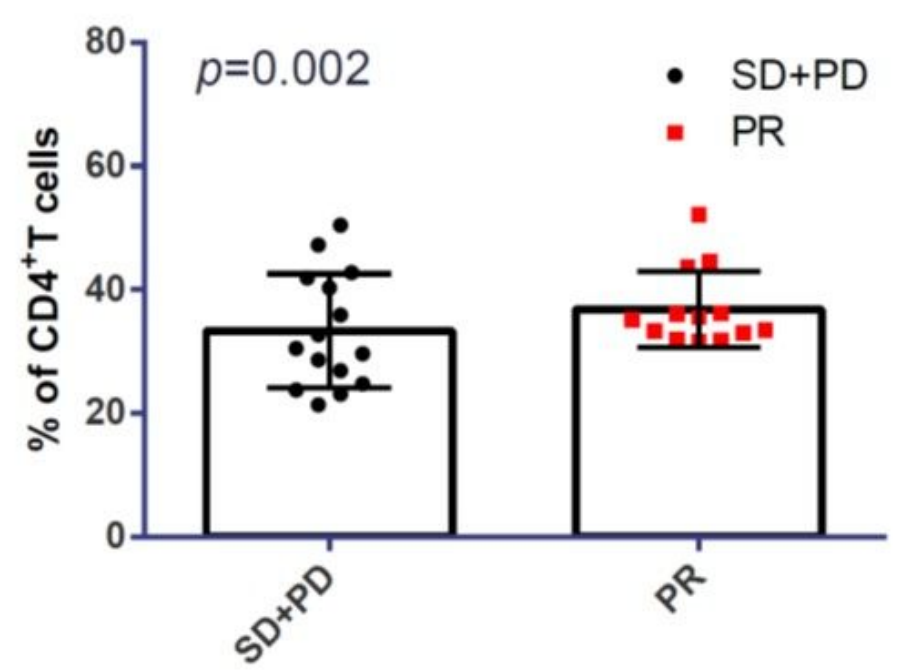

$\mathrm{CD}^{+} \mathrm{T}$ cells, Baseline

C

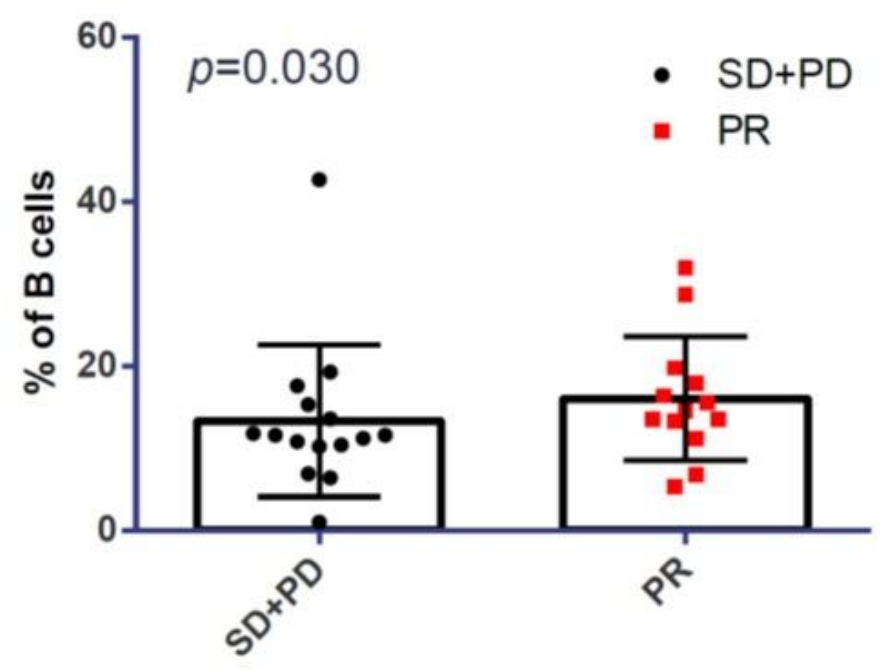

B cells, Baseline b

$\mathrm{CD}^{+} \mathrm{T}$ cells, Baseline

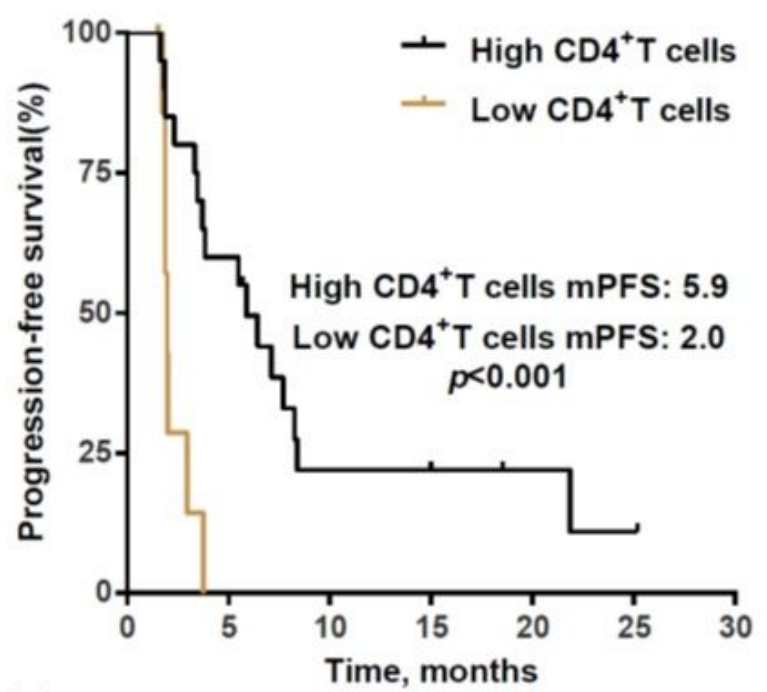

Number at risk

$\begin{array}{lllllll}\text { High } \mathrm{CD4}^{+} \mathrm{T} \text { cells } & 20 & 12 & 4 & 3 & 2 & 1 \\ \text { Low } \mathrm{CD4}^{+} \mathrm{T} \text { cells } & 8 & 0 & 0 & 0 & 0 & 0\end{array}$

d

B cells, Baseline

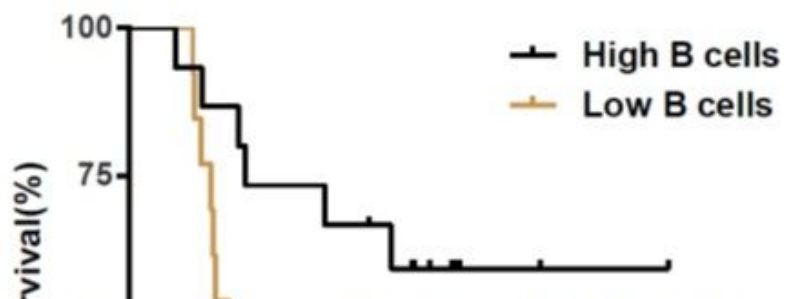

High B cells mOS: not reached Low $B$ cells mOS: 4.6 $p=0.030$

Number at risk

$\begin{array}{lrrrrrr}\text { High B cells } & 15 & 13 & 10 & 5 & 1 & 1 \\ \text { Low B cells } & 13 & 5 & 4 & 2 & 1 & 0\end{array}$

Figure 5

Associations of blood immune cell subpopulations and clinical outcomes. a Baseline proportion of CD4+ $T$ cells in responders and non-responders; $b$ Progression-free survival stratified by baseline CD4+ T cells level; c Baseline proportion of B cells in responders and non-responders; d Overall survival stratified by baseline B cells level.

\section{Supplementary Files}


This is a list of supplementary files associated with this preprint. Click to download.

- FigureS1.tif

- FigureS2.jpg

- FigureS3.jpg

- SupplementaryTable1.docx 